\title{
émulations
}

\section{À la recherche d'une muséographie internationale dans une nation globale}

\author{
La construction du nouveau Musée national d'art \\ contemporain de Corée du Sud dans les années 1980 \\ Ilmin Nah
}

Émulations - Revue de sciences sociales

$2018, n^{\circ} 26$, «Repenser la dichotomie 'national vs international' »

\section{Article disponible à l'adresse suivante}

https://ojs.uclouvain.be/index.php/emulations/article/view/nah

\section{Pour citer cet article}

Nah Ilmin, " À la recherche d'une muséographie internationale dans une nation globale ». Émulations, № 26, pp. 89-104. Mise en ligne le 15 décembre 2018.

DOI : 10.14428/emulations.026.07

Distribution électronique : Université catholique de Louvain (Belgique) : ojs.uclouvain.be

(C) Cet article est mis à disposition selon les termes de la Licence Creative Commons Attribution, Pas d'Utilisation Commerciale 4.0 International. http://creativecommons.org/licenses/by-nc/4.0/

Éditeur : Émulations - Revue de sciences sociales / Presses universitaires de Louvain https://ojs.uclouvain.be/index.php/emulations

ISSN électronique : 1784-5734

PUL PRESSES

UNIVERSITAIRES

DE LOUVAIN 


\title{
À la recherche d'une muséographie internationale dans une nation globale
}

\author{
La construction du nouveau Musée national \\ d'art contemporain de Corée du Sud \\ dans les années 1980
}

\begin{abstract}
IImin $\mathrm{Nah}^{1}$
[Résumé] Au cours des trois dernières décennies, les musées du monde entier n'ont eu de cesse de se penser par rapport à des dynamiques internationales, qui contribuent en partie à les renouveler. En Corée du Sud, le développement économique et l'ouverture internationale du pays dès les années 1980 - parallèlement à la tenue des Jeux olympiques (1988) - a été un puissant facteur de transformation du paysage muséal. Depuis lors, les musées sud-coréens se sont lancés dans une série de projets d'internationalisation, adoptant activement les normes modernistes occidentales. Alors que ce phénomène s'est intensifié via un processus de mondialisation croissante, on ne sait finalement que peu de choses sur l'histoire et la politique identitaire des musées sud-coréens. Centré sur le projet du nouveau Musée national d'art des années 1980, l'article mettra en lumière l'interaction dynamique entre le national et l'international ayant présidé à ce projet, participant ainsi au processus symbolique d'une réimagination de la Corée modernisée et globalisée.
\end{abstract}

Mots-clés : Musée national d'art ; art contemporain ; Corée du sud ; Séoul ; identité ; nation; jeux olympiques.

[Abstract] In the last three decades, museums around all over the world were engaged in increasingly complex international dynamics, which partly led to renew and reinvent themselves. South Korea's opening to the international environment, economic growth, and the Seoul Olympic Games (1988) were all powerful catalysts from the 1980 s for the transformation of national museum landscape. Thereafter, South Korean museums, whose activities and programs were so far based on national traditions and structures began to redefine themselves, adopting the so-called "modernist international" standards. While this phenomenon intensifies in the current age of cultural consumption and globalization, few literatures studied the evolution and the identity politics of the South Korean museums in relation to the country's internationalization movement. Focusing on the remaking of the National Museum of Contemporary Art (MMCA) in the 1980s, this article highlights the dynamic interaction between the national and the international dimensions of the project, participating in the symbolic reimagination of nation as global player.

Keywords: National Museum of Art; Contemporary Art; South Korea; Seoul; Identity; Nation; Olympic Games.

\footnotetext{
${ }^{1}$ Université de Kookmin et Université de Kyungsung, Corée du sud.
} 


\section{Introduction}

Depuis la fondation des premiers musées modernes en Occident au XVIIIe siècle, le musée, par son statut d'institution culturelle et patrimoniale, est susceptible de créer des valeurs contribuant à fixer des représentations collectives à l'échelle nationale et internationale. En particulier, le musée national d'art est le lieu où l'on sélectionne les œuvres d'art en raison de leur valeur esthétique et de leur sens historique commun. En patrimonialisant ainsi les œuvres choisies, il dessine les contours de l'identité nationale, créant « une communauté politique imaginaire et imaginée » (Anderson, 1991).

D’un autre côté, au cours des trois dernières décennies qui les ont vus se consolider dans un contexte de mondialisation accélérée, les musées du monde entier n’ont eu de cesse de se penser par rapport à des dynamiques internationales de plus en plus complexes qui contribuent en partie à les renouveler et les redéfinir. De ce point de vue, le musée représente sans doute, plus qu'aucune autre activité et production culturelle moderne, l'un des meilleurs objets d'interprétation afin de saisir la manière dont l'identité nationale a été construite et dont le processus d'internationalisation s'est achevé au sein d'une société, notamment dans les pays asiatiques.

En Corée du Sud, après avoir assuré la croissance économique et pendant que le pays préparait son entrée réussie au rang des pays développés via sa participation active dans la communauté internationale, la tenue des Jeux asiatiques (1986) et olympiques (1988) à Séoul a été un puissant facteur de transformation du paysage muséal. Au début des années 1990, le nombre de musées (nationaux, régionaux et privés) a lui-même pratiquement triplé en l'espace d'un an, passant de 131 en 1991 à 390 en 1992 (Lim², 1993). De plus, à l'instar de la tenue de la Biennale internationale de Gwangju (première biennale artistique internationale en Asie) en 1995, plusieurs grandes manifestations artistiques internationales, ainsi que des musées privés et publics, sont nés et ont grandi en différents endroits du pays, démontrant tout le dynamisme muséal de cette période qui allait encore s'accroître à l'avenir. Aujourd'hui, comme le démontre le hanllyu (ou vague coréenne) traversant le globe de nos jours, les arts et la culture de la Corée du Sud connaissent un succès international retentissant, notamment via ses produits culturels populaires tels que le cinéma, les séries télévisées et la K-pop, ainsi que la politique nationale de rayonnement de sa culture sur la scène internationale (Hong-Mercier, 2007).

Toutefois, malgré cet intense et spectaculaire développement culturel et des musées en Corée au cours des dernières décennies, dans les travaux de recherches internationaux publiés jusqu'à nos jours, peu d'études ont pris en compte de manière approfon-

\footnotetext{
${ }^{2}$ La transcription des termes et des noms propres coréens suit dans l'ensemble le système du Revised Romanization of Korean (Romanisation révisée, adoptée officiellement par le ministère de la Culture, des Sports et du Tourisme de Corée du sud depuis 2000) à l'exception de ceux déjà connus sous une autre orthographe.
} 
die l'évolution des musées coréens en relation avec le mouvement d'internationalisation du pays, pas plus qu'au sein des discours sur l'identité nationale. La raison principale est sans doute liée au caractère récent du phénomène, qui n’a débuté qu’à la moitié du XX $\mathrm{XX}^{\mathrm{e}}$ siècle et aussi parce qu'il n'existe pour ainsi dire aucun précédent au niveau des courants internationaux de recherches permettant de structurer le sujet. En effet, en Corée du Sud, ce n'est qu'à la fin des années 1990 que des travaux de recherches liés à l'historiographie des institutions muséales du pays ont été produits, formant ainsi un nouveau champ de recherches en sciences humaines et sociales. Après avoir consolidé la démocratie et la croissance économique (et sous la pression de la société civile), on a constaté de la part de la communauté intellectuelle et scientifique sud-coréenne une prise de conscience grandissante quant à la nécessité d'élaborer l'histoire des institutions culturelles et artistiques du pays ${ }^{3}$.

Partant de ce constat, c'est en nous focalisant sur le projet de construction du nouveau Musée national d'art contemporain de Corée du Sud (MMCA) ${ }^{4}$ dans les années 1980 que nous étudierons dans cet article l'origine, l'historique et la spécificité du processus d'internationalisation de cette institution coréenne. Après sa fondation en 1969 sous le régime militaire de Park Chung-hee, le MMCA a connu plusieurs phases de renouvellement et de relocalisation avant de se fixer dans son emplacement actuel, c'està-dire réparti sur trois sites au centre de Séoul et dans la ville de Gwacheon. Parallèlement à son site principal (ouvert en 1986), situé dans la ville de Gwacheon, qui se charge de la recherche et de l'exposition portant sur l'histoire de l'art contemporain coréen, l'institution possède aujourd'hui deux autres antennes : le musée de Séoul (ouvert en 2013) consacré à la promotion de l'art contemporain international par le biais d'actifs échanges internationaux et l'ancien Palais royal de Deoksu, où se trouvent les manifestations de l'art coréen autour de la période dite " moderne ", c'est-à-dire jusqu'à l'avant-guerre de Corée (1950). Nous questionnerons ici spécifiquement pourquoi et comment ce musée s'est engagé durant les années 1980 dans la construction de ce nouveau site à Gwacheon, en banlieue sud de Séoul, et quels changements lui ont succédé. Notre objectif principal sera, à partir d'une perspective à la fois sociohistorique et critique, de montrer l'interaction dynamique entre le national et l'international ayant

\footnotetext{
${ }^{3}$ Dès la fin des années 1990, plusieurs auteurs coréens ont publié des travaux retraçant de manière approfondie la vie et l'évolution des musées d'art en Corée, en particulier celles du MMCA à différentes périodes, depuis sa naissance jusqu'à la fin des années 1990. Par exemple : (Lee, 1998 ; Choi, 2002 ; Jeong, 2003).

${ }^{4}$ En mai 2013, avec l'ouverture du nouveau site au centre de Séoul, le Musée national d'art contemporain de Corée ou MOCA (appellation anglaise : National Museum of Contemporary Art) a été rebaptisé MMCA (National Museum of Modern and Contemporary Art, Korea). Notre travail portant essentiellement sur la période des années 1980-90, nous avons fait le choix de conserver l'appellation originale : Musée national d'art contemporain de Corée du Sud.
} 
présidé à ce projet, et donc la particularité et la complexité de sa portée symbolique, au regard de l'histoire et de l'identité nationales.

\section{Genèse et historique des institutions muséales en Corée et du Musée national d'art contemporain de Corée du Sud (MMCA)}

Si dans les pays occidentaux, la construction des premiers musées publics s'est généralement inscrite dans l'histoire du passage du régime monarchique à celui d'état-nation au XVIIIe siècle, l'étude de l'histoire des premiers musées coréens est à mettre en très étroite relation avec celle de l'histoire mouvementée du pays (Poulot, 1997; Lee N. Y., 1986). En effet, l'émergence de l'idée moderne du musée en Corée a débuté vers la fin du XIX ${ }^{e}$ siècle, quand le pays a été confronté à l'expansion de l'hégémonie occidentale et de l'impérialisme japonais sur son territoire. La Corée, qui jusqu'alors cherchait à s'isoler afin de maintenir son indépendance vis-à-vis de toute ouverture et influence de l'étranger, est donc passée par une grande période de transformations en s'ouvrant petit à petit à la modernité occidentale. C'est également durant cette période que les concepts occidentaux d'exposition et de musée en tant qu'institutions visuelles modernes ont été introduits au sein de la nouvelle génération des élites réformistes de gaehwa (la pensée des lumières), qui s'efforçaient d'apprendre et de s'approprier de nouveaux savoirs modernistes occidentaux.

En 1908, par ordre du roi Sunjong, le Musée impérial de la dynastie, premier musée du pays, a été ouvert (accompagné d'un jardin botanique et d'un zoo) dans le palais de Changgyeonggung à Séoul afin de présenter et de faire partager les collections royales aux visiteurs du palais. Or, dans le contexte d'une Corée alors ravagée par les luttes de factions sous l'influence des grandes puissances étrangères, le destin de ce premier musée coréen sera profondément enraciné dans une histoire riche en revirements : la colonisation japonaise (1910-1945), puis la libération (1945), l'occupation américaine (19451948), la formation de la République de Corée (1948) et, enfin, la guerre civile entre Corée du Sud et Corée du Nord (1950-1953).

En 1911, le Musée impérial de la dynastie a été rétrogradé et a changé son nom pour devenir le Musée de la famille du Prince Yi, suite à la signature d'un traité d'annexion de la Corée en août 1910 annonçant le début de l'occupation japonaise sur le territoire coréen. Le Japon impérial dont l'objectif était l'anéantissement total de l'histoire et de la culture coréennes a édifié en 1915, dans le cadre des stratégies coloniales de domination, le bâtiment du Musée du Gouvernement général japonais dans le palais de Gyeongbok, ainsi qu'un autre bâtiment dans le Palais de Deoksu afin d'y abriter un espace d'exposition d'art japonais. Afin de pouvoir remplir ces nouveaux musées voués

\footnotetext{
${ }^{5}$ Quelques articles en anglais (Choi S., 2011 ; Kal, 2011 ; Lee, 2011) ont exploré brièvement l'évolution du Musée national ou du Musée national d'art contemporain en relation avec le nationalisme postcolonial coréen.
} 
à « un savoir fondamental à la connaissance des territoires conquis », le Japon a effectué des recherches sur les tombes royales et les sites archéologiques du pays (Nanta, 2015). L'anarchie et le pillage ont ainsi détruit de nombreux lieux historiques durant cette période. En 1938, des œuvres d'art traditionnel appartenant au Musée de la famille du Prince Yi ont été transférées dans le musée d'art japonais du Palais de Deoksu, qui a ensuite pris le nom de Musée des Beaux-Arts de la famille royale Yi (également connu sous le nom de Palais des Beaux-Arts de Deoksu). Les collections du Musée du gouvernement général japonais et celles du Musée de la famille royale Yi ont donc constitué l'origine des futures collections du Musée national de Corée, créé en 1945, après l'indépendance du pays (Lee N.Y., 1986 ; Choi, 2002 ; Jeong, 2003 ; Lee, 2011 ; Jang, 2015).

Après la guerre de Corée et la division du pays, notamment suite à l'arrivée au pouvoir du général Park Chung-hee (par un coup d'État militaire) survenue le 16 mai 1961, la Corée du Sud a connu un processus d'industrialisation de plus en plus accéléré, notamment au travers du lancement de plans économiques quinquennaux. Afin d'évincer l'opposition et de prendre le contrôle du pays, le régime autoritaire de Park - qui a duré plus de quinze ans - s'est donné le rôle de " gardien de la culture nationale », imposant l'idéologie de l'ethno-nationalisme et de l'anticommunisme tout en s'appuyant sur la loyauté confucéenne selon laquelle le sacrifice de l'individu au profit du progrès et de la sécurité nationale va de soi (Cho, 2010). Le gouvernement a notamment fait de grands efforts pour sortir le pays du sous-développement, mettant en place une politique économique extrêmement protectionniste à l'égard des secteurs agricoles et industriels nationaux, tout en s'appuyant sur l'exportation de produits manufacturés, grâce au faible coût de la main-d'œuvre.

$\mathrm{Si}$, au cours de ce processus de reconstruction et de modernisation post-guerre, le développement économique a mobilisé toute l'attention de l'ensemble du pays (à savoir du peuple, des forces intellectuelles et gouvernementales), c'est également durant cette période qu'est né un regain d'intérêt concernant le besoin profond d'art et de culture, négligés pendant près d'un siècle de bouleversements. Dès la fin des années 1960, le gouvernement sud-coréen s'est donc mis à formuler et à appliquer un plan de développement à long terme pour la promotion de la culture et des arts afin de créer « une nouvelle culture nationale " perpétuant et mettant encore plus en valeur le patrimoine culturel et artistique (Kim, 1976 : 18). Il a alors décidé de prendre des mesures spécifiques en faveur de la culture, dont les plus importantes étaient l'élaboration d'un premier dispositif juridique, complété par la loi pour la promotion de la culture et des arts (1972) et la construction d'une Fondation pour la promotion de la culture et des arts coréens (1973).

C'est dans ce contexte spécifique des débuts en matière de politique culturelle nationale que le premier Musée national d'art contemporain a ouvert ses portes en 1969, dans un petit bâtiment au sein du palais royal de Gyeongbok, à Séoul. Toutefois, ce musée qui a été inauguré au moment où le gouvernement militaire a fait adopter par 
le Parlement un amendement constitutionnel permettant à Park de solliciter un troisième mandat présidentiel (confirmé par un referendum très surveillé) ne disposait d'aucune collection constituée au préalable, alors qu'il s'agit de la condition fondamentale et préalable à toute institution muséale. Conçu avant tout comme institution de légitimation d'un pouvoir politique illégitime, durant ses premières années de vie, le musée a été essentiellement utilisé comme salle d'événement pour être loué aux diverses associations artistiques et culturelles de l'époque, ou encore pour accueillir le Salon national organisé par le ministère de la Culture et de l'Information, représentant l'art officiel. Il a donc dû composer avec les limites qui lui étaient inhérentes (le manque de moyens d'intervention et de personnels qualifiés et formés en histoire de l'art et muséologie, la difficulté d'accès aux informations et aux recherches internationales sous la forte censure du gouvernement, sans oublier les conditions muséographiques incongrues du vieux bâtiment de l'ancien Palais royal, etc.) et ce, même après son déménagement au Palais de Deoksu en 1973, dans le système politique autoritaire et collectiviste qui a caractérisé la Corée du Sud jusqu’à la fin des années 1970 (Lee, 1998 ; Jeong, 2003).

\section{Les Jeux olympiques de Séoul et la construction du nouveau Musée national d'art contemporain à Gwacheon (1986) : créer de nos mains un grand musée international d'art}

Suite à l'assassinat de Park Chung-hee par son bras droit Kim Jae-gyu (chef des services de renseignements du KCIA) en octobre 1979, le général Chun Doo-hwan a pris le pouvoir le 12 décembre 1979 par un coup d'État militaire, tout comme son maître Park, deux décennies plus tôt (Chung, 2001). Parallèlement au contexte politique instable et perturbé du pays, sur le plan économique, les années 1980 ont marqué un tournant pour l'économie sud-coréenne : grâce à sa stratégie d'insertion dans l'économie mondiale, initiée dès les années 1970, vers la fin des années 1980, avec un P.I.B. total supérieur à 60 milliards de dollars, la Corée du Sud est en effet passée de la catégorie des pays à faible revenu (qui vivaient de l'aide internationale dans les années 1950) à celle des pays à revenu intermédiaire, devenant même l'une des nations les plus développées en Asie $^{6}$.

C'est dans ce nouveau contexte de transformation de la Corée du Sud en tant qu'actrice importante du commerce international, et notamment en tant que puissance exportatrice, que le gouvernement sud-coréen s'est engagé à construire un nouveau musée national d'art contemporain dans la ville de Gwacheon, en banlieue sud de Séoul.

\footnotetext{
${ }^{6}$ Voir «Économie et culture : le cas de la Corée du Sud », compte-rendu du déplacement d'une délégation du groupe sénatorial d'amitié France-Corée du Sud, 8-15 mars 2004. En ligne, consulté le 21 janvier 2018. URL : http://www.senat.fr/ga/rapport_coree/rapport_coree0.html
} 
Si ce projet a été, d’une part, le fruit de longs efforts déployés par la communauté artistique coréenne pour ériger un musée national d'art contemporain digne de ce nom dédié à la vie artistique contemporaine du pays, il s'est inscrit, d'autre part et avant tout, dans la politique de la préparation aux Jeux asiatiques de 1986 et, plus encore, aux Jeux olympiques de 1988 à Séoul. En effet, l'organisation de ces méga événements sportifs internationaux va toujours de pair avec l'embellissement des villes et la rénovation urbaine, ouvrant ainsi la voie à la modernisation et à l'internationalisation du pays. Elle sert généralement d'agent de transformation de tous les aspects (sociaux, politiques, économiques et culturels) de la nation (Kang, 2010). Le cas de la Corée du Sud n’y a pas fait exception, car il s'agissait de moments décisifs dans l'histoire du pays au cours desquels s'est imposée une nouvelle identité nationale.

Après l'arrivée au pouvoir du général Chun, l'ensemble du pays a été marqué par de forts mouvements politiques et sociaux contestataires, opposés à la pérennisation « désastreuse » de la dictature en Corée du Sud. Les critiques contre le gouvernement militaire ont rapidement augmenté, tant à l'échelle nationale qu'internationale, surtout après les mesures antidémocratiques sanglantes du régime contre la protestation des étudiants et des citoyens dans la ville de Gwangju, en mai 1980. Les soulèvements récurrents ont atteint leur point culminant en juin 1987, notamment après l'annonce du décès d'un étudiant (Park Jong-chul) au mois de janvier, sous la torture de la police et que cherchait à dissimuler le gouvernement (Chung, 1997: 25).

Dans ce contexte politique intérieur sinistré, l'administration de Chun a vu de nombreux avantages à l'idée d'accueillir les Jeux asiatiques et olympiques à Séoul. Tout d'abord, au niveau national, le gouvernement serait en mesure de promouvoir le fait que la Corée du Sud puisse enfin faire son entrée dans les rangs des pays du «premier monde » en s'alignant sur d'autres pays occidentaux, industrialisés et développés. Ensuite, au niveau international, cela conduirait la Corée à établir des liens avec d'autres pays étrangers avec lesquels elle n'avait jusqu'alors pas de relations diplomatiques, en particulier les pays communistes et les nations émergentes d'Asie du Sud et d'Afrique, qui constitueraient les futurs débouchés de ses produits manufacturés exportés. Il s'agissait donc d'une occasion en or afin de promouvoir et de montrer au reste du monde les riches ressources politiques, économiques et culturelles du pays, ce dernier devenant un membre actif des institutions internationales. Le nouveau gouvernement militaire pouvait ainsi établir sa légitimité politique (Kang, 2010 : 546-548).

Après avoir obtenu en 1981 à Baden-Baden, en Allemagne, l'organisation des Jeux olympiques de 1988, le chef de l'État a exprimé à plusieurs reprises sa volonté d'être en mesure d'en faire " les Jeux olympiques de la culture » et de répondre ainsi aux exigences d'ouverture et d'internationalisation de l'époque (Chun, 1983:50-51). En particulier, la consolidation de l'identité nationale par la voie du renforcement des équipements culturels est devenue l'un des objectifs centraux des politiques culturelles de la Ve République. Le chiffre du budget de l'État dans le domaine de la culture et des arts a donc triplé en quelques années : les dépenses culturelles ont triplé entre 1981 et 1987, 
avec 14284 millions de wons, chiffre qui a été multiplié par trois après 1987 (47 423 millions de wons 7 ). De plus, le régime s'est engagé à transformer en profondeur le paysage de Séoul, comme si la ville était le visage du pays. Il s'est notamment consacré à renforcer de nombreuses infrastructures urbaines (nouveaux stades sportifs, aéroports, hôtels, transports en commun, etc.) répondant aux normes internationales établies par les imageries urbaines des grandes métropoles occidentales comme New York, Paris, Berlin, Londres, etc. En outre, une majeure partie du budget a été dépensée pour de grands travaux présidentiels faisant la part belle à la culture : en témoignent la construction du Seoul Art Center (1984), du nouveau site du Centre de musique traditionnelle de Corée (1987), ainsi que la transformation de l'ancien bâtiment du gouvernement général japonais pour abriter le Musée national de Corée (1986).

C'est donc dans cette ambiance frénétique de politique d'innovation culturelle et urbaine des années 1980 qu'est né le projet de construction du nouveau Musée national d'art contemporain. Dès 1981, le ministère de la Culture et de l'Information a mis en place les projets d'études préalables pour ce nouveau musée, à Gwacheon. Celui-ci a été conçu pour offrir un «nouvel espace culturel » au nombre croissant de personnes de la classe moyenne urbaine du pays s'installant au sud de la capitale (Hong, 2011 : 94-97). L'ensemble de ce plan architectural s'est donc inscrit dans le cadre du projet plus large de construction du Seoul Grand Park, un grand complexe de divertissements et de loisirs bordé par le Seoul Horse Race Park (ouvert en 1989), se composant lui-même d'un zoo et d'un parc d'attractions. Le comité de pilotage du projet a confié la conception du bâtiment à l'architecte Kim Tai-soo (qui à l'époque travaillait aux États-Unis, après des études d'architecture à l'Université de Yale ${ }^{8}$ ). Ainsi, c'est avec l'objectif de " créer un musée d'art du monde de nos propres mains » que les travaux de construction ont officiellement été lancés le 1er mai 1984.

\footnotetext{
${ }^{7}$ Cette hausse du volume des dépenses est confirmée par la volonté du gouvernement de consacrer à la culture une part sans cesse croissante de son budget : 0,18 \% en 1981 et $0,30 \%$ en 1987. Voir (Yang-yeul,1995).

${ }^{8}$ Le projet architectural de Kim Tai-soo a été retenu le 4 décembre 1982, à l'issue d'une compétition fermée contre celui de Kim Swoo-geun. Ce dernier est un éminent architecte coréen qui a réalisé beaucoup de projets architecturaux gouvernementaux jusqu'aux années 1980 , parmi lesquels le stade olympique de Séoul (1987).
} 


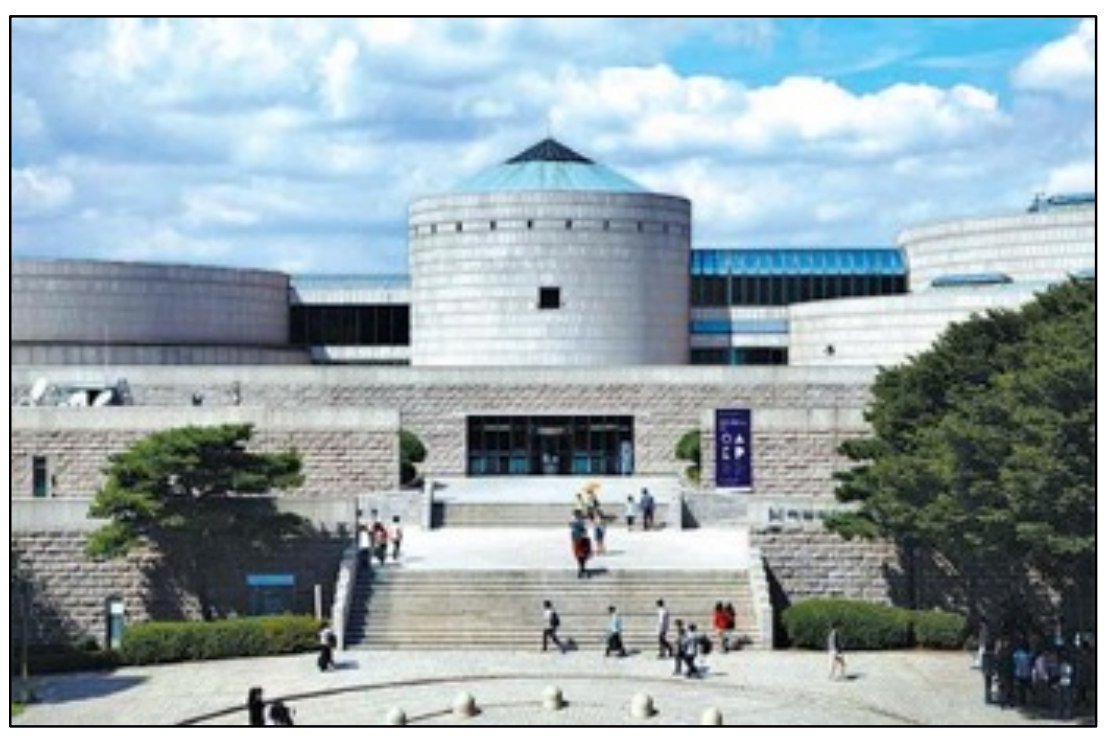

Figure 1. Musée national d'art contemporain de Corée, Gwacheon. (Photographie (c) MMCA Kim Yong Kwan)

\section{Le nouveau Musée national d'art contemporain de Corée du Sud, nouveau visage d'une nation globalisée?}

Après deux ans et demi de travaux de construction pilotés par Daewoo Construction et avec un budget atteignant les vingt milliards de wons', le nouveau musée s'est enfin ouvert au public le 25 août1986, soit un mois avant les Jeux asiatiques de Séoul. Bâti au sein d'un immense parc de sculptures (33000 mètres carrés), le musée est un bâtiment semi-ovale construit en granit coréen comprenant six salles d'exposition réparties sur trois étages. Son ouverture s'est accompagnée d'une cérémonie d'inauguration en présence du couple présidentiel et d'un grand nombre d'hommes politiques et de personnalités du monde artistique et culturel coréen. Les médias nationaux lui ont également octroyé une large couverture et ont encouragé les gens à visiter le Musée national d'art nouvellement né.

\footnotetext{
${ }^{9}$ À l'époque, environ 25 millions de dollars américains, ou 250 millions de francs français.
} 


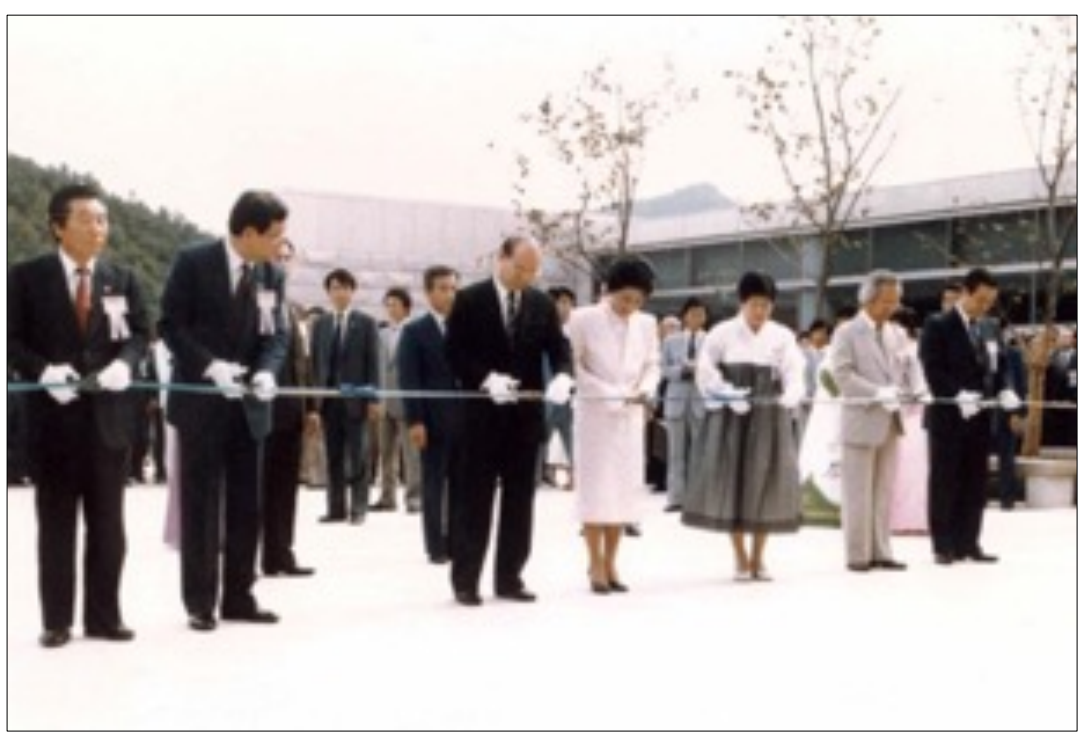

Figure 2. Cérémonie d'inauguration du nouveau Musée national d'art contemporain de Corée en présence du couple présidentiel Chun Doo-hwan et Rhee Soon-ja, le 25 août 1986, Gwacheon, Corée du Sud.

(Photographie @ MMCA Art Research Center, Gwacheon)

Quels sont alors les enjeux symboliques et opératoires de la nouvelle muséographie du MMCA par rapport à la précédente ? Si l'on compare le nouveau musée au précédent, il faut d'emblée souligner le renouvellement de son orientation vers un véritable musée d'art contemporain et international. Même si la forme extérieure de l'édifice s'inspire d'une forteresse et d'un phare coréens traditionnels, vu de l'intérieur, l'adoption du modèle moderniste de white cube dans les salles d'exposition ou de la méthode d'accrochage neutre (suivant les normes muséographiques internationales issues du modèle du musée moderniste, comme le MoMA à New York), ainsi que la présence d'une rampe hélicoïdale de plus de 200 mètres de long formant le hall d'entrée du musée (qui rappelle naturellement le rotunda de Frank Lloyd Wright au musée Guggenheim à New York, parangon de l'architecture moderniste internationale) montrent qu'au centre de cette conception, on trouve surtout la recherche d'une nouvelle muséographie internationale. De plus, l'architecture du musée est mieux adaptée à ses activités et ses fonctionnements muséologiques, avec plusieurs salles dédiées aux expositions temporaires et à l'accrochage des collections permanentes, des parcours muséographiques modulables, l'équipement des réserves pour garantir les meilleures conditions de conservation des œuvres, etc. La conception du bâtiment du nouveau musée marque donc une 
rupture avec celle du traditionnel et statique style d'architecture de l'ancien palais royal qui a précédé.

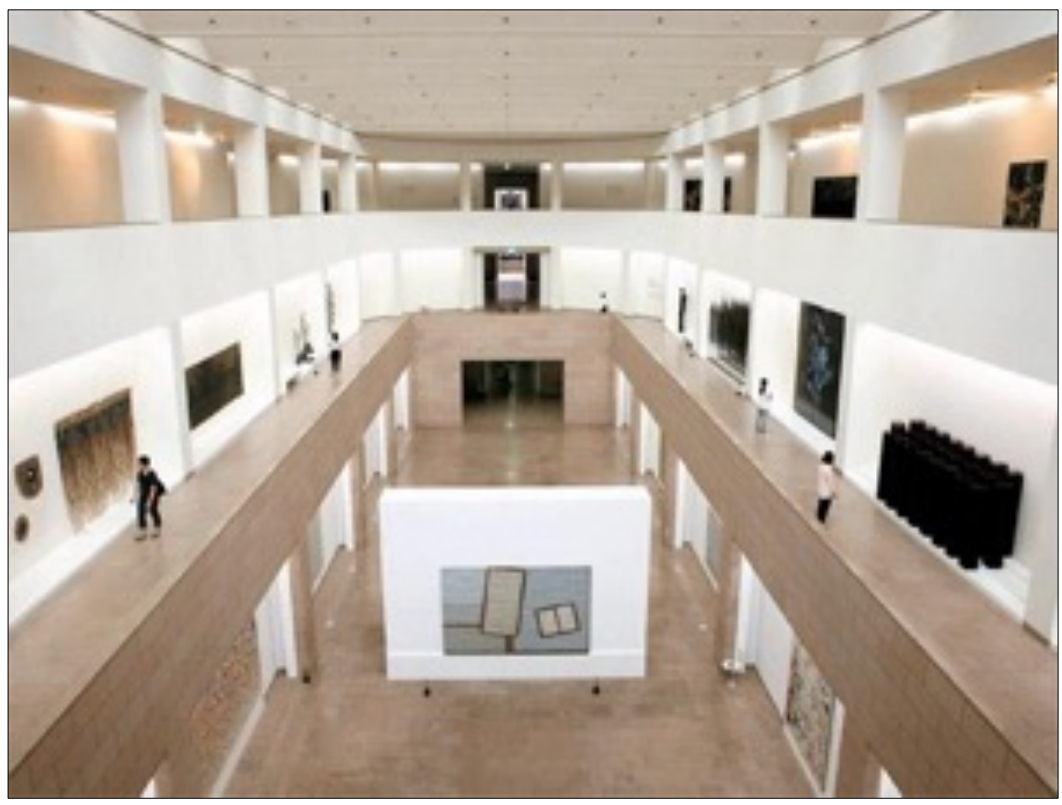

Figure 3. Vue du hall central reliant les salles d'exposition. (Photographie @ MMCA Kim Yong Kwan)

Par ailleurs, d'un point de vue organisationnel, l'institution connaît aussi une série de réformes. D’abord, l'établissement, à caractère public dans son ensemble, sera géré selon la tradition du service public, alors que le corps de l'administration du musée est constitué non seulement par des personnalités appartenant à la fonction publique, mais aussi par des hommes recrutés pour leurs qualités professionnelles. Ce système privilégie notamment la possibilité d'engager un expert comme Lee Kyung-sung (éminent critique et historien d'art coréen du XX $\mathrm{XX}^{\mathrm{e}}$ siècle) pour diriger le musée. Ce dernier réaffirme ensuite son mandat lié à sa mission sociale en tant qu'institution culturelle moderne : mise en place du département d'éducation assurant le nouveau rôle de l'instruction publique, professionnalisation du personnel et de gestion plus rationnelle... Enfin, le musée adopte une politique active d'acquisitions en matière d'art contemporain international suite à la constitution du comité d'Achat, en 1984. Un simple survol des noms des artistes dans les collections acquises durant cette période permet de constater la forte place qu'occupe la création artistique contemporaine et étrangère (en particulier euro-américaine) en son sein : de nombreuses œuvres d'artistes internationaux 
tels que Joseph Beuys, Claude Viallat, Andy Warhol, Robert Rauschenberg, Georg Baselitz, Jörg Immendorff, Marcus Lüpertz et Nam June Paik ont ainsi été intégrées au sein de la collection, sans parler des regroupements constitués grâce aux œuvres des principaux acteurs de la scène nationale contemporaine (Lim, 2005).

Si le principe et le modèle des musées modernistes internationaux ont été largement répandus et respectés dans l'organisation du nouveau musée, de tels programmes se manifestent clairement dans le récit véhiculé lors des expositions inaugurales du musée. Lors de son ouverture, quatre expositions temporaires ont ainsi accueilli les visiteurs : la première, Art of the world today : l'art contemporain 86 Séoul/Asie, montrait les œuvres d'artistes venant des pays d'Asie participant aux Jeux asiatiques de Séoul (Sri Lanka, Malaisie, Japon, Népal, Singapour, Inde, Indonésie, Thaïlande, Hong Kong, etc.). La deuxième, intitulée L'art français du XXe siècle, donnait à voir l'art français jusqu'aux années 1950, avec entre autres les peintures et sculptures d'artistes tels que Matisse, Soulages, Dubuffet et Simon Hantaï. Dans le cadre de la troisième exposition, Frederick R. Weisman Art Collections, le musée montrait plusieurs œuvres des principaux représentants majeurs du Pop Art américain, issues de la collection de ce grand collectionneur d'art américain. Enfin, la quatrième exposition, L'Art contemporain coréen : hier et aujourd'hui, représentait l'évolution de la pratique picturale coréenne du $\mathrm{XX}^{\mathrm{e}}$ siècle, depuis la période de la colonisation japonaise jusqu'à nos jours, au travers de plus de 700 œuvres exposées, afin de montrer « comment la Corée du Sud avait pu se réconcilier avec la modernité, s'écartant de son triste passé du fait de nombreuses guerres, ou bien des invasions étrangères », comme le souhaitait à l'époque le directeur du musée dans le catalogue de l'exposition (Lee K. S., 1986 ; Kee, 2009).

L'ensemble de ce projet d'exposition, organisé en étroite collaboration avec les plus hautes instances culturelles des pays concernés, se caractérisait donc par sa dimension à la fois internationaliste et nationaliste. En alignant les œuvres coréennes avec les chefs-d'œuvre majeurs internationaux, le caractère « national » de l'art était largement souligné (Hong, 2011 ; Lee, 2011). Les gens visitant à l'époque le nouveau musée ont été invités à percevoir le lieu comme le site international des énergies de la création artistique contemporaine. Ces expositions ont même exhorté les Coréens à éprouver un sentiment de fierté et d'appartenance à une nation jouant désormais un rôle actif dans la production et la diffusion des tendances artistiques internationales. Le but était non seulement de façonner une certaine idée de l'histoire mondiale de l'art, mais aussi de susciter chez le public coréen une interrogation anticipée sur le rôle et la place désormais occupés par la Corée dans la nouvelle cartographie de l'art mondial. Toutefois, comme l'ont souligné de nombreux critiques de l'époque, malgré l'abondance des œuvres montrées, aucune œuvre contemporaine coréenne de minjung, que l'on peut traduire par «l'art du peuple », n'avait été autorisée. En effet, ces images étaient inspirées de traditions populaires ou folkloriques dont l'origine était, en grande partie, une réaction aux mesures violentes du gouvernement envers le mouvement de démocrati- 
sation (Kee, 2009; Hong, 2011). Autrement dit, par leur dimension engagée et dénonciatrice, elles étaient susceptibles d'apporter des idées importunes dans le monde imaginaire et idéalisé créé par la politique du régime.

Si l'on considère que le musée et le patrimoine artistique sont au cœur du schéma de modernisation culturelle et constituent aussi un modèle de la cohésion nationale, un tel manque de points de vue critiques quant aux programmes du nouveau Musée national d'art contemporain nous amène finalement à réfléchir sur le principe idéologique sous-jacent du gouvernement militaire : pour ce dernier, l'art et la culture sont considérés comme les lieux de la promotion du progrès et de l'avancement de l'intérêt national, ce qui lui permet d'acquérir également sa légitimité politique. Dans ce cadre, la mise en œuvre d'un grand musée d'art national, rempli d'œuvres d'art internationales, semble être tout à fait naturelle afin de révéler le nouveau visage de la Corée, tant au niveau national qu'international : un pays tourné vers le monde et l'avenir, qui soit un acteur principal au sein de la communauté internationale, au travers de sa modernité culturelle et de sa visibilité mondiale.

\section{Conclusion}

Comme tous les autres musées du monde, en tant que reflet de la vie sociale et du paysage culturel au sein des organisations gouvernementales, le MMCA garde les traces matérielles du passé et du présent artistique du pays, qui aujourd'hui vont au-delà de la dichotomie entre national et international. La construction du nouveau site du MMCA, entamée dès les années 1980 a marqué en ce sens une nouvelle ère pour cette institution incarnant l'histoire tumultueuse du pays : le musée a été reconstruit au moment précis où la Corée du Sud s'engageait dans une politique de plus en plus libérale et internationale, laquelle a ensuite culminé dans les années 1990 par le discours institutionnel de segyehwa (globalisation ${ }^{10}$ ) qui a balayé le pays. Par la suite, la Corée du Sud s'est efforcée de devenir " première de classe mondiale » (segyeillyu), de se joindre à l'hégémonie occidentale et d'être un membre économique actif du G20, de l'OCDE, etc. Cette étude souligne que le Musée national d'art fonctionnait lui-même dans ce cadre idéologique comme un « média » public, véhiculant et soutenant la politique nationale afin d'imaginer la modernité culturelle dans une nation globale.

Certes, de tels paradigmes de l'internationalisation adoptés par le gouvernement sudcoréen dès les années 1980 et les lieux de rencontre internationaux qu'il a créés dans l'espace muséal et public ont permis d'enrichir et d'élargir les perspectives des artistes et du grand public, remodelant progressivement le paysage artistique et muséal coréen.

\footnotetext{
${ }^{10}$ On a choisi le terme de « globalisation » plutôt que celui de « mondialisation » pour essayer de distinguer le sens perçu en coréen de celui véhiculé en français par le mot «mondialisation » : en coréen, le terme segyehwa est connoté comme « intégration au modèle occidental », notion évidemment absente en français.
} 
Or, comme l'on a vu, dans la société coréenne des années 1980 où l'intérêt national et l'intégration internationale ont été primordiaux, mais aussi où l'on a constaté un grand mouvement populaire de résistance contre une compréhension monolithique de la culture nationale, la voix des individus et des groupes minoritaires et locaux a finalement été peu intégrée dans les discours officiels du musée. Dans le contexte actuel et global où les idées et les informations circulent en temps réel et de façon de plus en plus accélérée, la recherche d'une modernité et d'une internationalité ne signifie pas la même chose qu'auparavant pour les institutions culturelles sud-coréennes. L'ouverture du nouveau site du musée dans le centre de Séoul en novembre 2013 a représenté à cet égard un nouvel élan pour cette institution muséale coréenne qui ambitionne d'être désormais le nouveau « hub » artistique de l'Asie. Reste donc à voir, dans une société coréenne en permanente mutation, où le progrès technologique est roi, comment le nouveau MMCA va pouvoir imaginer l'identité et l'avenir du pays.

\section{Bibliographie}

ANDERSON B. (1991 [1983]), Imagined Communities. Reflections on the Origin and the Spread of Nationalism, Londres/New York, Verso.

Cно H-Y. (2010), Dong'wondoen geundaehwa (Mobilised Modernisation), Séoul, Humanitas.

CHor S. (2011), « Re-thinking Korean cultural identities at the national museum of Korea », in Simon Knell, Peter Aronsson, Arne Bugge (dir.), National Museums: New studies from around the world, London/New York, Routledge, 2011, p. 290 301.

CHOI Y. (2002), « Deoksugung misulgwanui yeoksawa gungnipgeundaemisulgwan gusang » (L'histoire du Musée des Beaux-Arts de Deoksu et la conception du Musée national d'art moderne), Art \& Museum Studies, $n^{\circ} 13$. En ligne, consulté le 21 janvier 2018. URL : http://www.mmca.go.kr/study/study13/study13_b1.html.

Chun D.-H. (1983), Pour une nation d'avant-garde, Politique pour la Nouvelle année : exposé du président, Séoul, Service coréen d'information pour l'Étranger.

Chung B., SeIzelet E. (1997), « La démocratisation à l'épreuve en Corée du Sud », Revue d'études comparatives Est-Ouest, vol 28, nº 3, p. 23-42.

Chung B. (2001), « Politique et religion en Corée », Revue d'études comparatives EstOuest, vol. 32, $n^{\circ} 1$, p. 85-110.

HoNG-Mercier S-K. (2007), « Les études culturelles et le phénomène de Hallyu (Korean Wave) en Asie de l’Est », Médiation et information, n²4-25, p. 165-174. 
Hong K. (2011), Aesthetic Constructions of Korean Nationalism: Spectacle, Politics and History, Londres/New York, Routledge.

JANG S-H. (2015), A representation of nationhood: The National Museum of Korea, thèse de doctorat en philosophie, Leicester, University of Leicester.

JEONG J-M. (2003), « Hang'uk geun,hyeondaemisulgwansa yeong'u : Gungnipmisulgwane daehan insikgwa jedojeok mosunui geunwoneul jungsimeuro » (L'Étude de l'histoire du musée d'art moderne et contemporain en Corée : autour de l'idée du musée national d'art et les paradoxes de l'approche institutionnelle), Art \& Museum Studies, $n^{\circ} 14$. En ligne, consulté le 21 janvier 2018. URL : http://www.mmca.go.kr/study/study14/study14_a9.html .

KANG S-P. (2010), Korean Culture and Seoul Olympic Studies. Kang, Shin-pyo: His Olympics: the case of the Seoul Olympic Games, Séoul, The National Folk Museum of Korea.

KEE J. (2009), «Longevity studies : the contemporary Korean art exhibition at fifty », in Your Bright Future: 12 Contemporary Artists from Korea, Houston/Los Angeles, Los Angeles County Museum of Art/The Museum of Fine Arts, p. 17-29.

KIM Y-S. (1976), La Politique culturelle dans la République de Corée, Paris, Presses Universitaires de France/UNESCO.

LEE N-Y. (1986), « Les Musées de Corée », Museum International. UNESCO, nº 149, vol. 38 , $\mathrm{n}^{\circ} 1$, p. $30-35$.

LEE K-s. (1986), Hang'ukhyeondaemisurui eojewa oneul (Korean art today: The National Museum of Modern Art), Gwacheon, National Museum of Contemporary Art.

LEE I-B. (1998), Misulgwan jedo yeongu (Study on Museum Institution), Séoul, Korea National University of Arts.

LEE J-J. (2011), « The national museum as palimpsest : Postcolonial politics and the national museum of Korea », in S. KNell, P. ARonsson, A-B Amundsen (dir.), National Museums: New studies from around the world, Londres/New York, Routledge, p. 373-385.

LIM M-Y. (1993), « L'élaboration et l'utilisation des indicateurs culturels en Corée », Rapport pour l'UNESCO. URL http://unesdoc.unesco.org/images/0009/000942/094257fb.pdf

LIM D-G. (2005), « Gungnipyeondaemisulgwan sojangpum sujipjedoui hyeonhwanggwa gwaje » (L'État et la perspective du système de la constitution des collections du Musée national d'art contemporain), Art \& Museum Studies, $\mathrm{n}^{\circ} 16$. En ligne, $\begin{array}{lllll}\text { consulté } & \text { le } & 21 & \text { janvier } & 2018 .\end{array}$ 
https://www.mmca.go.kr/research/artInfoResearchList.do?menuId=3040000000

NANTA A. (2015), « L'organisation de l'archéologie antique en Corée coloniale (19021940) : du terrain aux musées coloniaux », Ebisu, vol. 52, p. 117-154.

OH Y-Y. (1995), « Hang'ugui munhwahaengjeongchegye 50nyeon » (50 years of Korean Cultural administration system), The Journal of Cultural Policy, vol.7, p. 29-74.

Poulot D. (1997), Musée, nation, patrimoine, 1789-1815, Paris, Gallimard. 